\title{
Multimodality imaging for preprocedural planning of percutaneous mitral valve repair: a comprehensive review
}

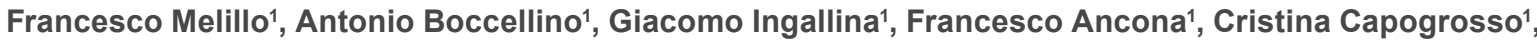 \\ Antonio Napolano', Stefano Stella ${ }^{1}$, Eustachio Agricola ${ }^{1,2}$ \\ ${ }^{1}$ Cardiovascular Imaging Unit, San Raffaele Hospital, Milan 20132, Italy. \\ ${ }^{2}$ School of Medicine, Vita-Salute San Raffaele University, Milan 20132, Italy.
}

Correspondence to: Prof. Eustachio Agricola, Head of Cardiovascular Imaging Unit, Cardio-Thoracic-Vascular Department, San Raffaele Hospital, Via Olgettina 60, Milan 20132, Italy. E-mail: agricola.eustachio@hsr.it

How to cite this article: Melillo F, Boccellino A, Ingallina G, Ancona F, Capogrosso C, Napolano A, Stella S, Agricola E. Multimodality imaging for preprocedural planning of percutaneous mitral valve repair: a comprehensive review. Mini-invasive Surg 2020;4:81. http://dx.doi.org/10.20517/2574-1225.2020.83

Received: 12 Aug 2020 First Decision: 18 Sep 2020 Revised: 28 Sep 2020 Accepted: 12 Oct 2020 Published: 6 Nov 2020

Academic Editor: Azeem Latib Copy Editor: Cai-Hong Wang Production Editor: Jing Yu

\begin{abstract}
New transcatheter mitral valve (MV) therapies are now available as alternatives to surgical and medical treatments in patients at high or prohibitive operative risk. Multimodality imaging including echocardiography, cardiac magnetic resonance, and cardiac computed tomography provide complementary information to guide patient and device selection. Morphology and functional anatomy of the MV should be carefully evaluated to determine the feasibility of percutaneous treatment; to identify the best therapeutic approach, either leaflet or annulus or combined; and to predict the probability of procedural success that is crucial for subsequent outcome and should be integrated by comprehensive preprocedural assessment of chamber size, biventricular systolic and diastolic function, valvopathy hemodynamic impact and aortic or peripheral vascular disease. The spectrum of transcatheter options is now wide and encompasses leaflet repair, direct or indirect annuloplasty, and cordal implantation. The aim of this review is to provide an overview on the role of multimodality imaging in the patient selection and preprocedural planning of percutaneous mitral valve repair.
\end{abstract}

Keywords: Transcatheter mitral intervention; 3D-echocardiography; multimodality imaging 


\section{INTRODUCTION}

The burden of clinically significant mitral valve disease is noteworthy in the elderly population and therapeutic options were constrained due to patients' high operative risk so far. Thanks to new transcatheter mitral valve (MV) therapies, alternatives to surgical and medical treatments are now available. Accurate patient selection is crucial for procedural success and is based on careful preprocedural multimodality imaging evaluation. Echocardiography, cardiac magnetic resonance (CMR), and cardiac computed tomography (CT) may provide complementary information to guide patient and device selection. Evaluation of mitral valve anatomy, identification of MV lesion, and quantification of defect severity should be integrated by comprehensive preprocedural assessment of chamber size, biventricular systolic and diastolic function, hemodynamic impact, and aortic or peripheral vascular disease. The aim of this review is to provide an overview on the role of multimodality imaging in the patient selection and preprocedural planning of percutaneous mitral valve repair.

\section{ECHOCARDIOGRAPHY}

Echocardiography is pivotal for diagnosis, anatomical and functional characterization, and quantification of mitral regurgitation (MR) severity. Transthoracic echocardiography (TTE) is the first level imaging modality and allows a comprehensive evaluation of valve disease, chamber size, and function. Evaluation of mitral and pulmonary flow pattern as well as pulmonary artery pressure and chamber dimensions are precious indexes of hemodynamic load secondary to the valvopathy. Transesophageal echocardiography (TEE) is a second level imaging modality, and it is usually indicated for a deep evaluation of valve anatomy and MR grading. 2D echo allows both morphological and functional evaluation of the MV: the former is based on detection of leaflet abnormalities such as thickness, redundancy, and calcification, as well as identification of annular calcification, and the latter is based on evaluation of systo-diastolic leaflet motion according to Carpentier classification and is fundamental to understand the disease etiology and to guide the therapeutic strategy. $3 \mathrm{D}$ echocardiography (3DE) provides an added value in detailing $\mathrm{MV}$ morphology and precise localization of pathology through $3 \mathrm{D}$ rendering, multiplanar reconstruction (MPR), and 3D color-Doppler. $3 \mathrm{D}$ rendering is pivotal for morphological assessment as it provides a more realistic representation of the MV, which can be visualized through several perspectives ${ }^{[1]}$. Moreover, $3 \mathrm{DE}$ is superior to $2 \mathrm{D}$ echo in detailing the lesions in terms of scallop disease and commissural involvement ${ }^{[2]}$ and localizing the calcifications and additional findings such as clefts or tissue deficiency. The MPR mode allows the assessment of annular dimensions and its dynamics during the cardiac cycle ${ }^{[3]}$, mitral valve area (MVA), and characterization of the disease (flail/prolapse detection, localization, and analysis) [Figure 1]. Furthermore, it allows studying specific sites of interest such as the potential grasping zone for transcatheter repair with leaflet approach in terms of measurement of posterior leaflet length, leaflet motion, and calcification/thickness [Figure 2].

The site of origin of the regurgitant jet may be identified by $3 \mathrm{D}$ color-Doppler, especially from the left ventricular perspective that directly shows the flow convergence area. Moreover, measurement of 3D vena contracta area is a new method for MR quantification showing higher accuracy compared to $2 \mathrm{D}$ colorDoppler, particularly in the presence of multiple or eccentric MR jets ${ }^{[4]}$. Color-coded 3D parametric maps may be created by either automatic or semiautomatic software, provide indices of MV remodeling, and may localize MV pathology ${ }^{[5]}$.

Finally, 3D transthoracic echo provides reproducible measurement of LV volumes and function with similar accuracy compared to $\mathrm{CMR}^{[6]}$.

Speckle tracking imaging represents a more sensitive tool to assess LV dysfunction than ejection fraction and may improve the selection of candidates for the procedure. Recently, baseline GLS value $<-9 \%$ has been 

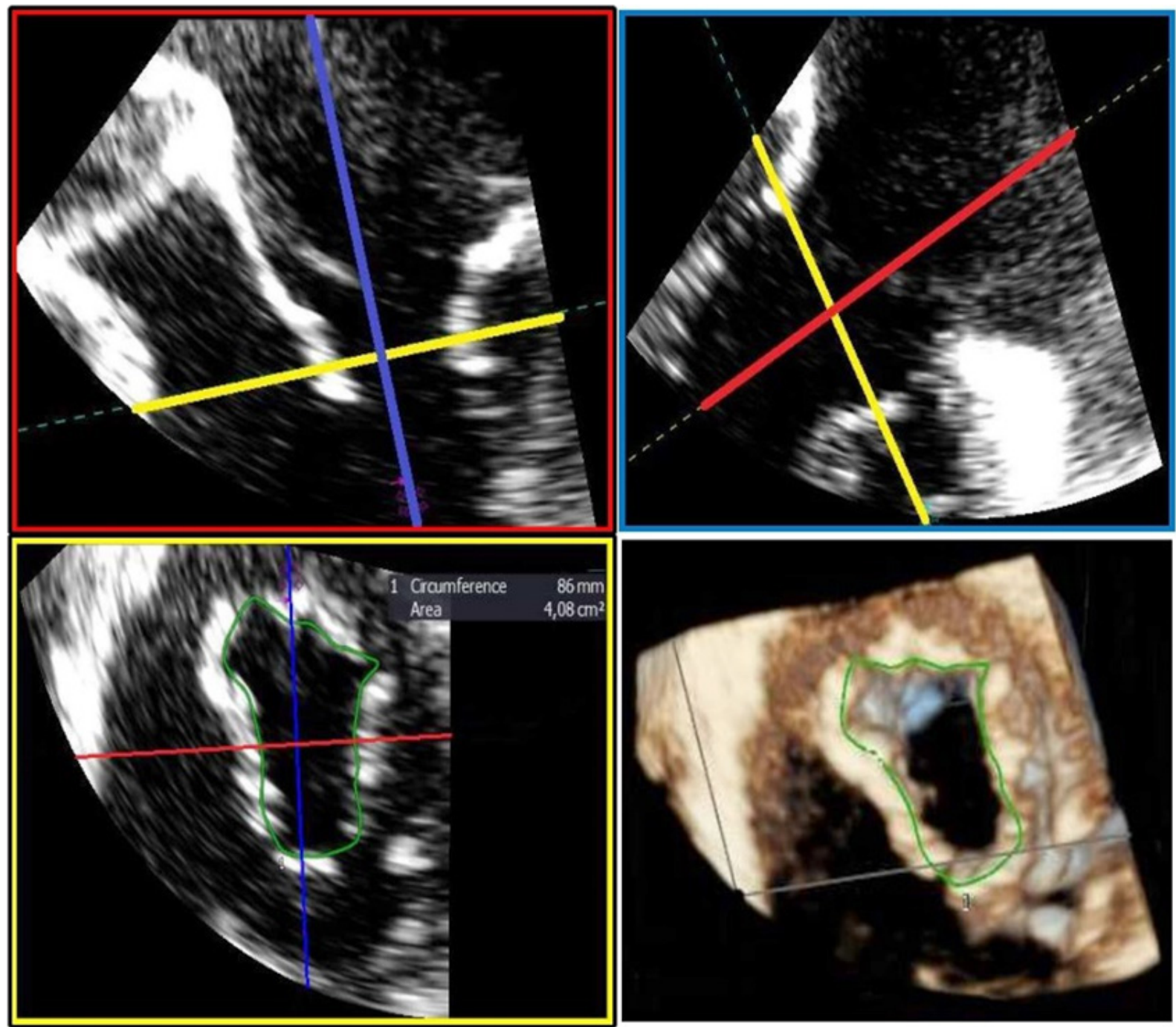

Figure 1. MPR analysis of 3D dataset to measure mitral valve area. The mitral valve is fully open. The tips of mitral leaflets are identified in the blue and red planes to delineate the orifice, allowing the measurement of the mitral valve area on the axial. MPR : multiplanar reconstruction

demonstrated to be an independent predictor of LV reverse remodeling and clinical outcomes in patients with secondary MR treated with MitraClip ${ }^{[7]}$.

Preprocedural evaluation of MR severity should be performed according to EACVI and ASE guidelines for native valve regurgitation ${ }^{[8,9]}$. Different methods are available such as qualitative (visualization of color-Doppler regurgitant jet area and evaluation of continuous wave Doppler signal), semi-quantitative (vena contracta width, mitral inflow velocity, and pulmonary vein flow pattern), or quantitative (effective regurgitant orifice area (EROA), regurgitant volume (RVol), and regurgitant fraction (RF) by PISA or volumetric method). As a true gold standard for accurate MR grading is not available, a multiparametric approach is the cornerstone for a reliable quantification of MR. Qualitative and quantitative parameters have different strengths and weaknesses that should be balanced to reduce their own limitations: jet area is linearly correlated with the driving pressure and may underestimate eccentric jets; vena contracta width measurement assumes the EROA to be circular; pulsed wave Doppler analysis of mitral and pulmonary flow is highly dependent on intra-chambers pressures and left atrial compliance; and PISA method is affected by time of regurgitation and shape of flow convergence area ${ }^{[9]}$. The quantitative assessment of 


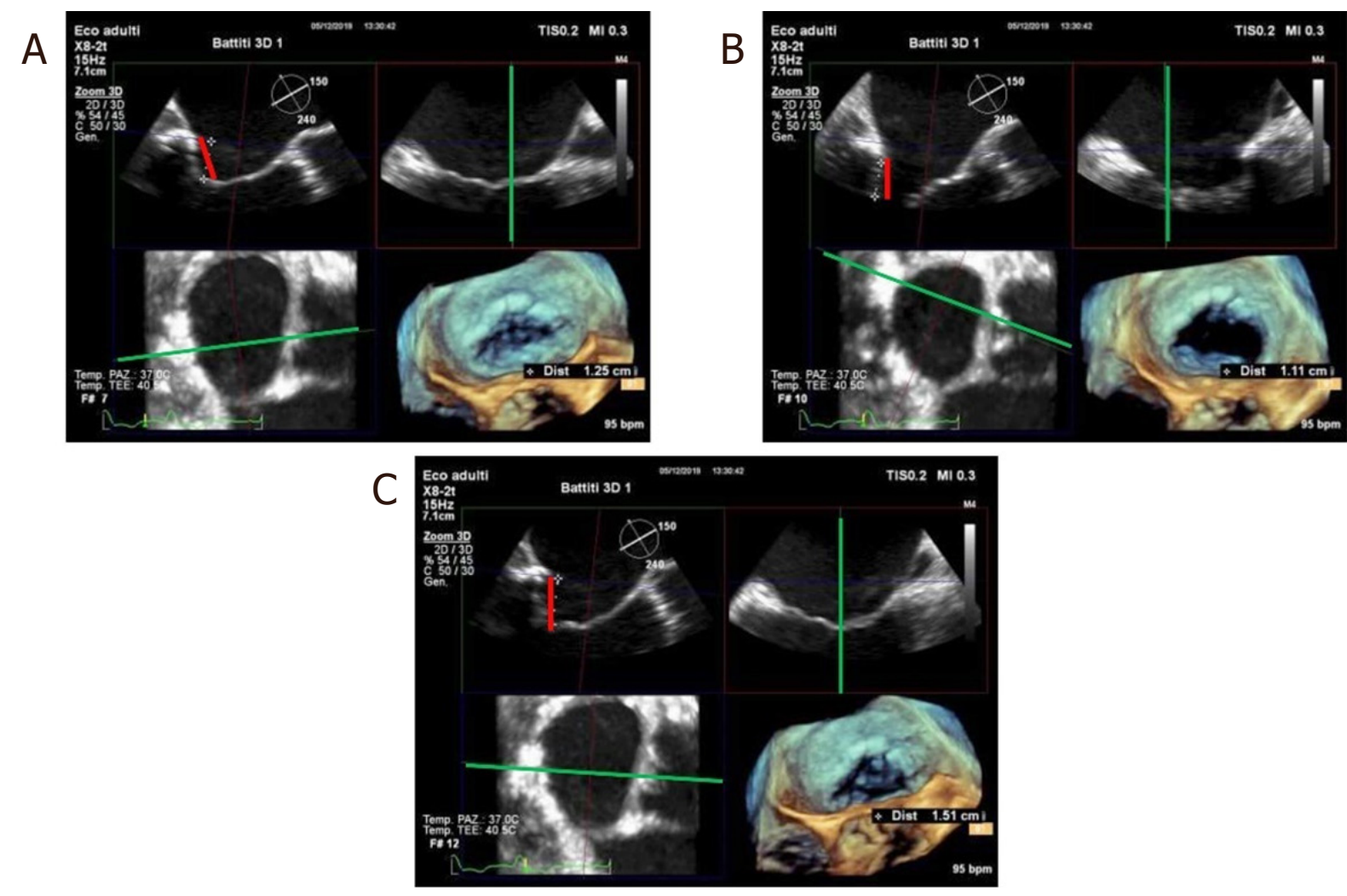

Figure 2. Usefulness of MPR method to measure the posterior leaflet length in specific area of interest identified by the green plane: (A) centro-lateral; (B) central; and (C) centro-medial. MPR : multiplanar reconstruction

secondary MR is further affected by the following conditions: the regurgitant orifice is often crescentshaped, the proximal convergence zone is irregular, jets could be multiple and eccentric, often shows a typical biphasic pattern, and systolic blunting of the pulmonary venous flow pattern may be influenced by the underlying cardiomyopathy.

The analysis of $3 \mathrm{D}$ vena contracta area (3D VCA) can overcome the geometric assumptions of $2 \mathrm{D}$ PISA method, and it has shown a good correlation with regurgitant volume calculated by CMR [Figure 3]. Moreover, the ability to correctly localize jets makes the 3D-VCA very useful when multiple MR jets are present and the measurement of EROA or VC from a single jet does not reflect the true regurgitant volume ${ }^{[10]}$. However, the optimal cut-off value of $3 \mathrm{D}$ VCA to define severe MR is still debated.

Finally, a careful evaluation of LV volumes, EF, and effective stroke volume is essential. Ventricles of different volumes can have similar EROAs but different RVol and RF. ASE and EACVI guidelines consider different thresholds to discriminate severe MR. An algorithm for quantitative assessment of FMR based on estimation of RF has been proposed to further stratify the patients among those within the grey area (EROA of 20-30 $\mathrm{mm}^{2}$ and a RVol of 30-44 mL), identifying patients with $\mathrm{RF}>50 \%$ those at high risk ${ }^{[11]}$.

Stress echocardiography may represent an added value in patients presenting with discordant symptoms and rest echocardiography, allowing to evaluate effort tolerance and variation in regurgitant volume during exercise $^{[1]}$. Experience on stress echo in the setting of percutaneous mitral valve repair is still limited and most evidence derives from studies on patients undergoing MV surgery: an exercise induced systolic pulmonary artery pressure $(\mathrm{sPAP})>60 \mathrm{mmHg}$, exercise induced right ventricular dysfunction, and a reduced LV contractile reserve (exercise increase in ejection fraction $<4 \%$ or increase in global longitudinal 

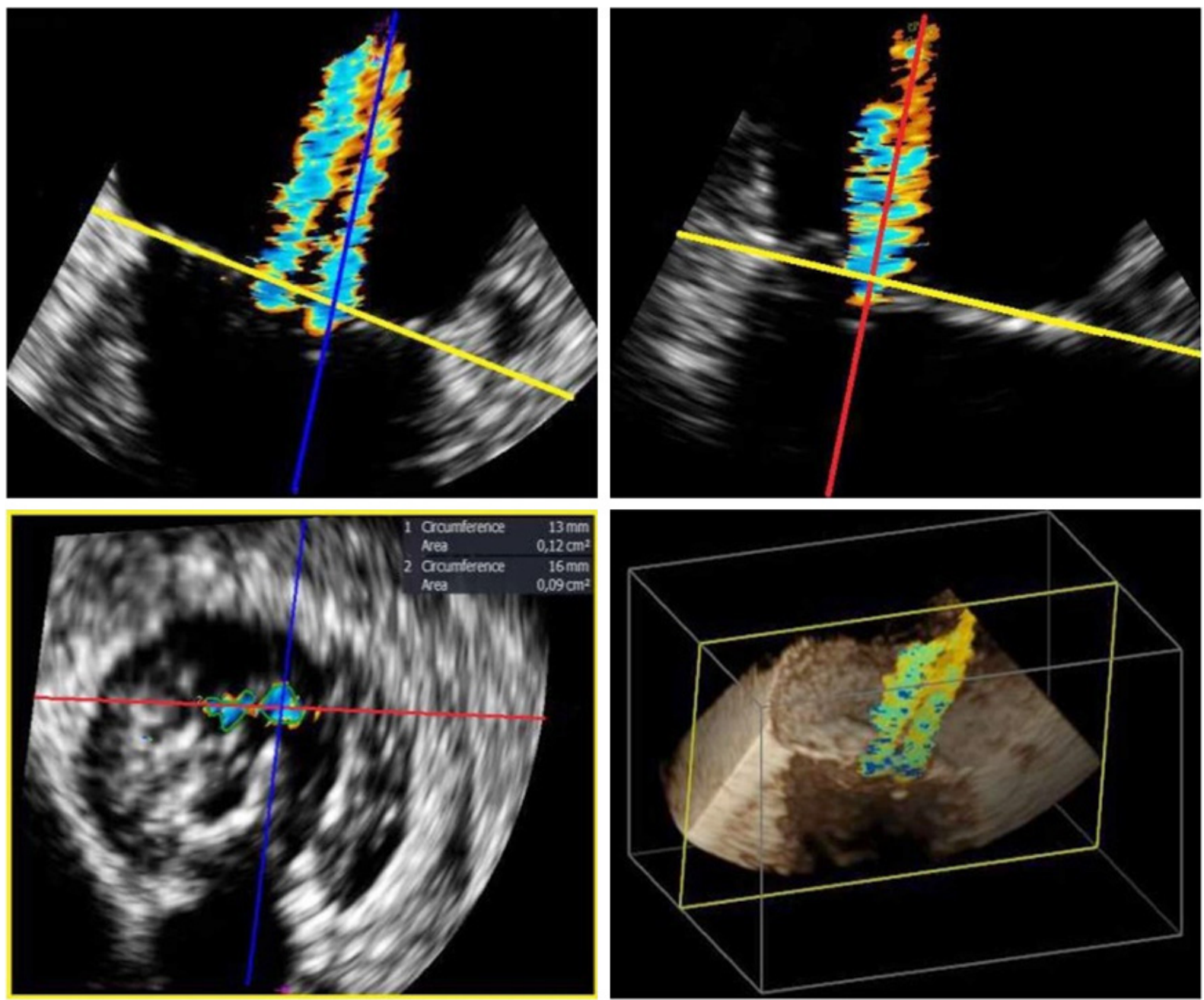

Figure 3. MPR reconstruction of the regurgitant jet. The narrowest portion of the jet close to the tip of the leaflets is identified and vena contracta area is measured on the axial plane (yellow). The measurement may be done for each regurgitant jet and the value. MPR: multiplanar reconstruction

strain $<2 \%$ ) have been identified as predictors of poor prognosis in degenerative $\mathrm{MR}^{[1]}$. In secondary $\mathrm{MR}$, an exercise increase in EROA $>0.13 \mathrm{~cm}^{2}$ and $\mathrm{sPAP}>60 \mathrm{mmHg}$ carry a poor prognosis ${ }^{[12]}$, while MR decrease with exercise because of improvement of walls motion, recruitment of ischemic segments, and ultimately reduction of the tethering forces could identify patients who would benefit from optimal medical therapy and revascularization prior to the correction of mitral disease ${ }^{[13]}$. On this basis, a study on 39 patients treated with MitraClip confirmed that a decrease in MR grade during stress echo was associated with limited clinical benefit from the procedure ${ }^{[14]}$.

\section{CARDIAC MAGNETIC RESONANCE}

$\mathrm{CMR}$ is the gold standard to assess cardiac dimension, function, and tissue characterization. It has acquired an emerging role in the context of MV as a reliable quantitative method to assess MR in discordant cases or when echocardiography is of poor quality. It can provide an effective quantification of MR, also in the contest of multivalvular disease, without limitations of imaging window or body habitus. The measurement of the Rvol requires two different imaging techniques: steady state free precession (SSFP) sequences to calculate left ventricular (LV) stroke volume from the difference between LV diastolic and systolic volumes and phase contrast sequences to measure the LV forward stroke volume ${ }^{[15]}$ [Figure 4]. RVol and RF are calculated as difference between LV total stroke volume and forward flow. 


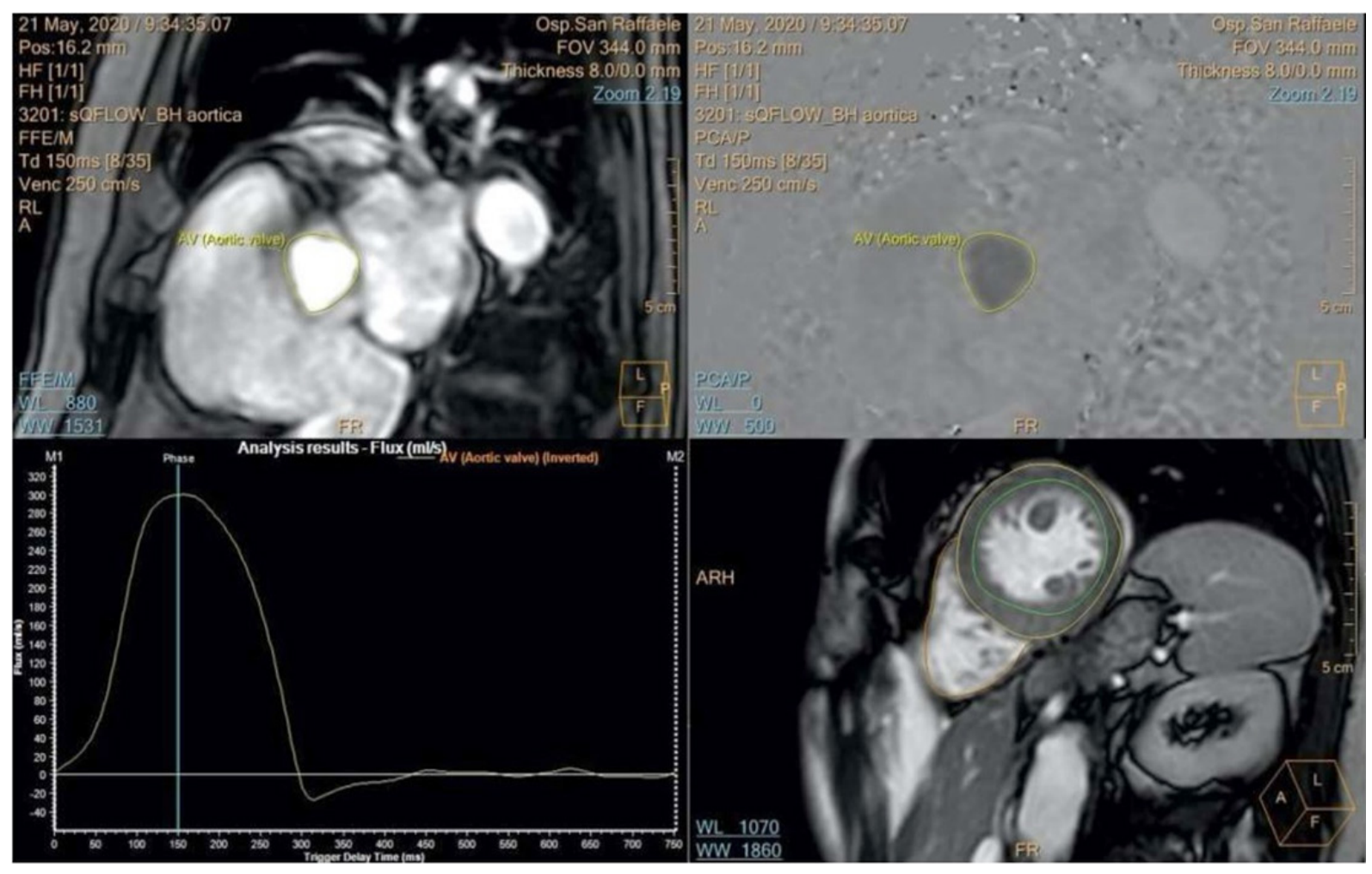

Figure 4. Quantification of mitral regurgitation by CMR. SSFP sequences to evaluate LV stroke volume from the difference between LV diastolic and systolic volumes (bottom right) and phase contrast sequences to measure the LV forward stroke volume (top). CMR: cardiac magnetic resonance; SSFP: steady-state free precession imaging; LV: left ventricle

The limits of CMR include the duration of the exam, the difficulties in identifying correctly the basal slice of LV in SSFP sequences, and the need to select the correct perpendicular slice to the ascending aorta with the correct velocity encoding setting in phase contrast sequences.

CMR was firstly compared to angiography with good correlation, however existing discordances in the assessment of regurgitation with transthoracic (TT) and transesophageal (TE) 2D/3D echocardiography remain even in presence of severe regurgitation ${ }^{[15,16]}$. In five studies ${ }^{[17-21]}$, the agreement between CMR and $2 \mathrm{D}$ echocardiography among patients diagnosed with severe regurgitation ranged between $20 \%$ and $66 \%$, with echocardiography usually showing more severe regurgitation than CMR. Studies comparing CMR with 3D echocardiography reported improved absolute agreement, but with wide limits of agreement, suggesting that the grading differences remain ${ }^{[22,23]}$.

In the upcoming years, the potential advancement of the $4 \mathrm{D}$-flow CMR retrospective valve tracking method, quantifying flow directly at mitral valve, could further improve CMR assessment ${ }^{[24]}$.

The thresholds that define severity in CMR might differ from echocardiography since the latter seems to quantify a larger RVol in the same patient ${ }^{[25]}$ and the actual recognized cut-offs for CMR are RVol $>55-60 \mathrm{~mL}$ and $\mathrm{RF}>40 \%{ }^{[17,26,27]}$.

Limited data are available comparing CMR and echo for detection of leaflet abnormalities.

A fundamental added value of CMR is considered the ability to accurately evaluate cardiac chamber function and dimensions ${ }^{[9]}$ and to characterize the extent of scarring and myocardial viability, which 

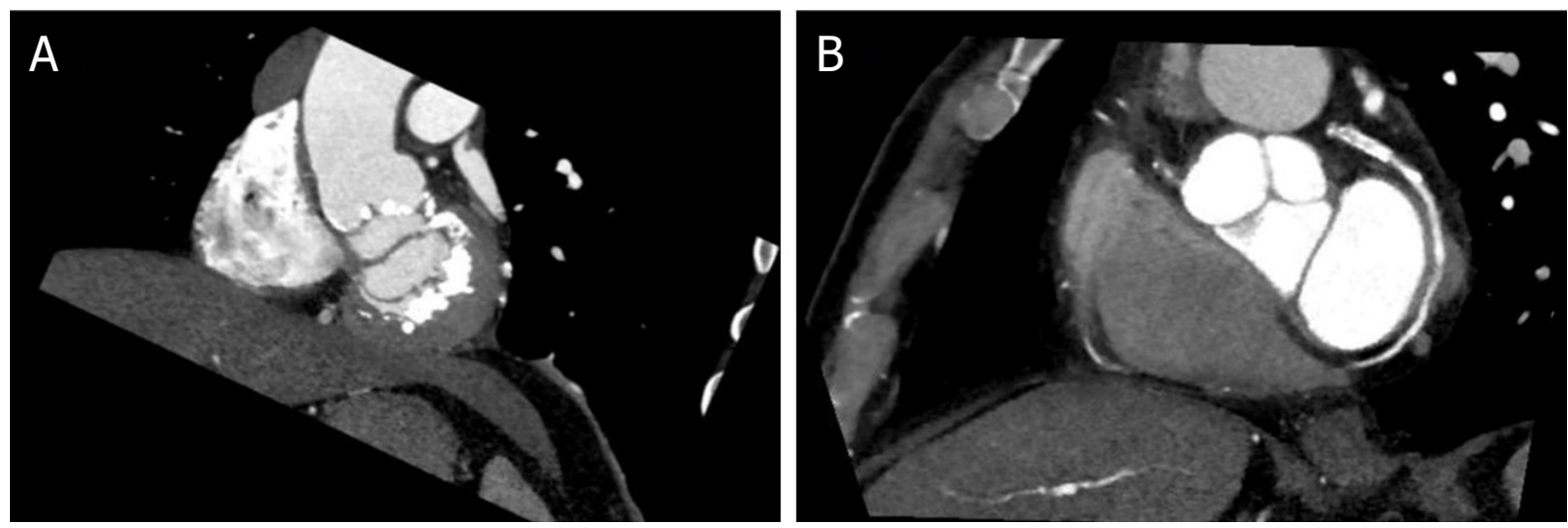

Figure 5. (A) Cardiac CT short-axis view of the mitral valve at the level of the mitral annulus, showing annular calcification; and (B) short axis view at the level of the atrioventricular groove showing the course of the left circumflex artery

provides further stratification over ventricular volumes ${ }^{[28]}$. In degenerative mitral valve prolapse, it allows easy detection and quantification of the mitral annular disjunction and assessment of LV posterior wall and papillary muscle fibrosis for arrhythmic risk stratification ${ }^{[29,30]}$.

Finally, CMR is useful to assess structural abnormalities of the MV apparatus, such as anomalous insertion of papillary muscle directly into the AML or hypertrophied and apically displaced anterolateral papillary muscle in hypertrophic cardiomyopathy ${ }^{[31]}$.

\section{COMPUTED TOMOGRAPHY}

Cardiac multidetector computed tomography (MDCT) has an excellent spatial resolution and is highly reproducible, being relatively operator-independent. On the other hand, the temporal resolution is inferior compared to echocardiography and MRI, and the quality of the exam is highly dependent on the arrhythmic burden. The technical suggestion for optimal analysis of MV apparatus is the retrospective ECG-gated acquisition of R-R interval from $0 \%$ to $90 \%$, in order to have all the datasets available for MPR and correction of arrhythmia-related artifacts. Moreover, to limit the artifacts and increase the temporal resolution, a CT scanner with 64 detector rows is recommended ${ }^{[32]}$. MDCT, thanks to its excellent bloodtissue interface and the high-spatial 3D imaging, provides a comprehensive visualization of cardiac and vascular structures and can give detailed information on mitral annular shape and sizing, valvular calcification, papillary muscles position and dimension, LV shape and dimension, and the relationship of the heart with chest wall. Furthermore, multiplanar and curved planar (CPR) reconstructions allow a comprehensive assessment of the course of coronary arteries and veins with respect to MV apparatus ${ }^{[33]}$.

MDCT is the gold standard for the precise location, extension, and objective quantification of calcifications [Figure 5]. The extent of calcifications into the annulus (MAC), the leaflets, and the subvalvular apparatus, as well as in the myocardium and left ventricle outflow tract, can be easily visualized. Finally, MDCT may play an emerging role in MV valve evaluation to determine MV area ${ }^{[34]}$, leaflet length, prolapse/flail parameters, tethering angles, and quantification of $\mathrm{MR}^{[35]}$.

\section{CHOICE OF TRANSCATHETER MITRAL VALVE REPAIR APPROACH}

Patients with significant mitral regurgitation may present comorbidities or technical challenges that increase surgical risk or contraindicate surgery. These patients, if symptomatic or requiring recurrent hospital admission for heart failure despite optimal guideline-directed medical therapy, represent candidates to percutaneous interventions. However, morphology and functional anatomy of the mitral valve should be 


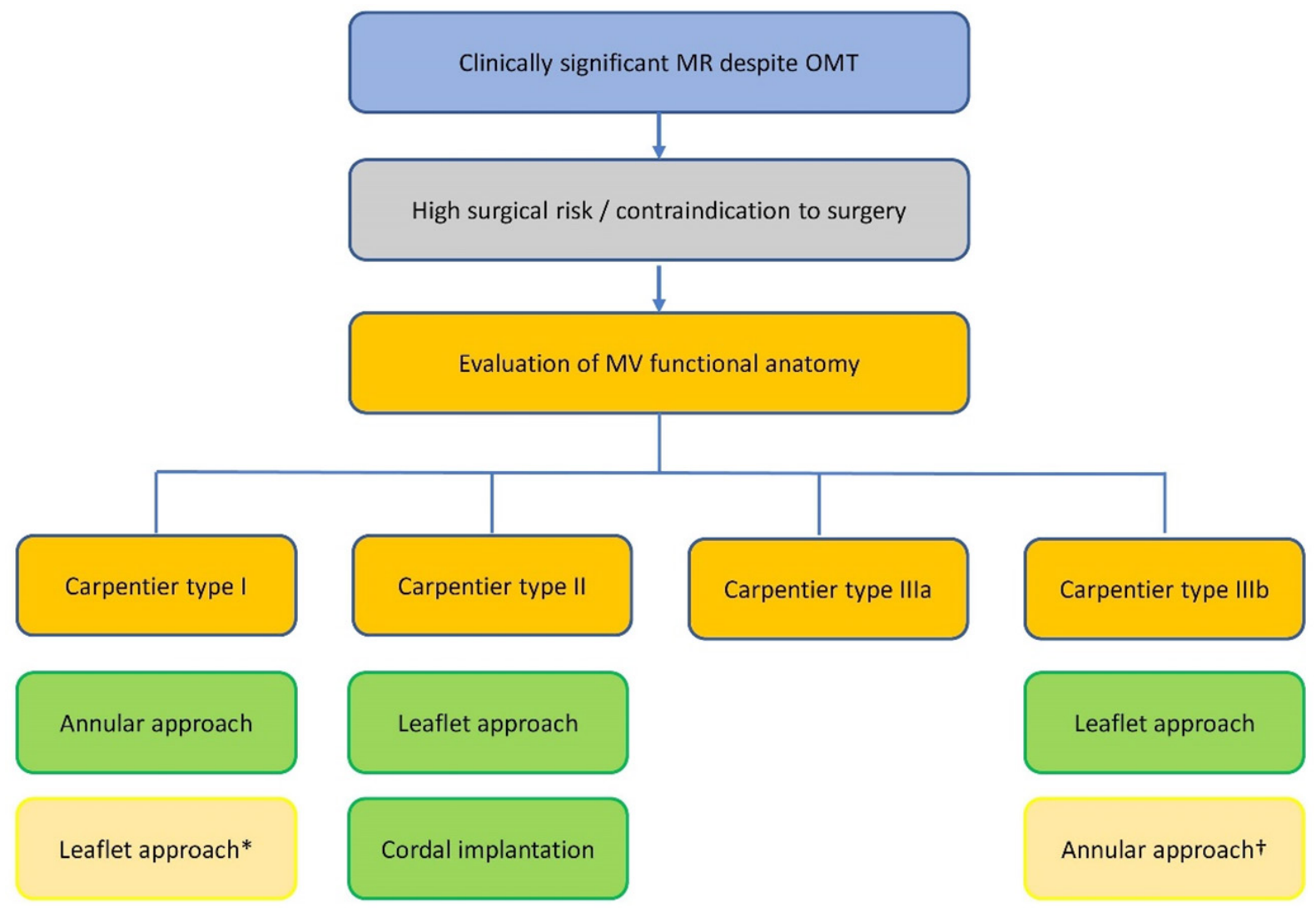

Figure 6. Spectrum of available transcatheter mitral valve repair options according to functional anatomy of the mitral valve. *Expected suboptimal result due to residual annular dilatation; $\uparrow$ ideal tethering should be limited (coaptation depth $<10 \mathrm{~mm}$ )

carefully evaluated to determine the feasibility of percutaneous treatment; to identify the best therapeutic approach, either leaflet or annulus or combined; and to predict the probability of procedural success that is crucial for subsequent outcome.

Both primary and secondary MR may be treated with a leaflet approach, while the annular approach is usually reserved for secondary MR. Functional classification helps to understand pathology and further guide therapeutic approach [Figure 6]: Carpentier Type I, normal leaflet motion and position (annular dilation, leaflet perforation, cleft); Type II, excess leaflet motion (prolapse, flail); Type IIIa, restricted leaflet motion in systole and diastole (rheumatic, fibro-calcification); and Type IIIb, restricted leaflet motion in systole (tethering secondary to ischemic or non-ischemic cardiomyopathy). In absence of specific contraindications, a leaflet approach is suitable for treating Types II and IIIb and suboptimal for Type I, while annular approach is most appropriate for Types I and IIIb if limited leaflet tethering is present.

\section{PREPROCEDURAL PLANNING FOR TRANSCATHETER MITRAL LEAFLET REPAIR}

Transcatheter leaflet repair is based on the surgical technique developed by Alfieri ${ }^{[36]}$. Two devices (MitraClip, Abbott, Illinois, USA; and PASCAL, Edwards Lifescience, California, USA) that allow reproducing the technique into a catheter-based approach are available thus far. The devices are equipped with two arms to grasp and approximate the free edges of anterior and posterior mitral leaflets.

The site origin of the jet represents the target lesion, so a preprocedural evaluation is firstly aimed at evaluating leaflet tissue quality, length, and mobility in the area of potential grasping zone. The analysis of the $3 \mathrm{D}$ dataset by MPR represents an added value for this purpose, allowing the evaluation of leaflet 


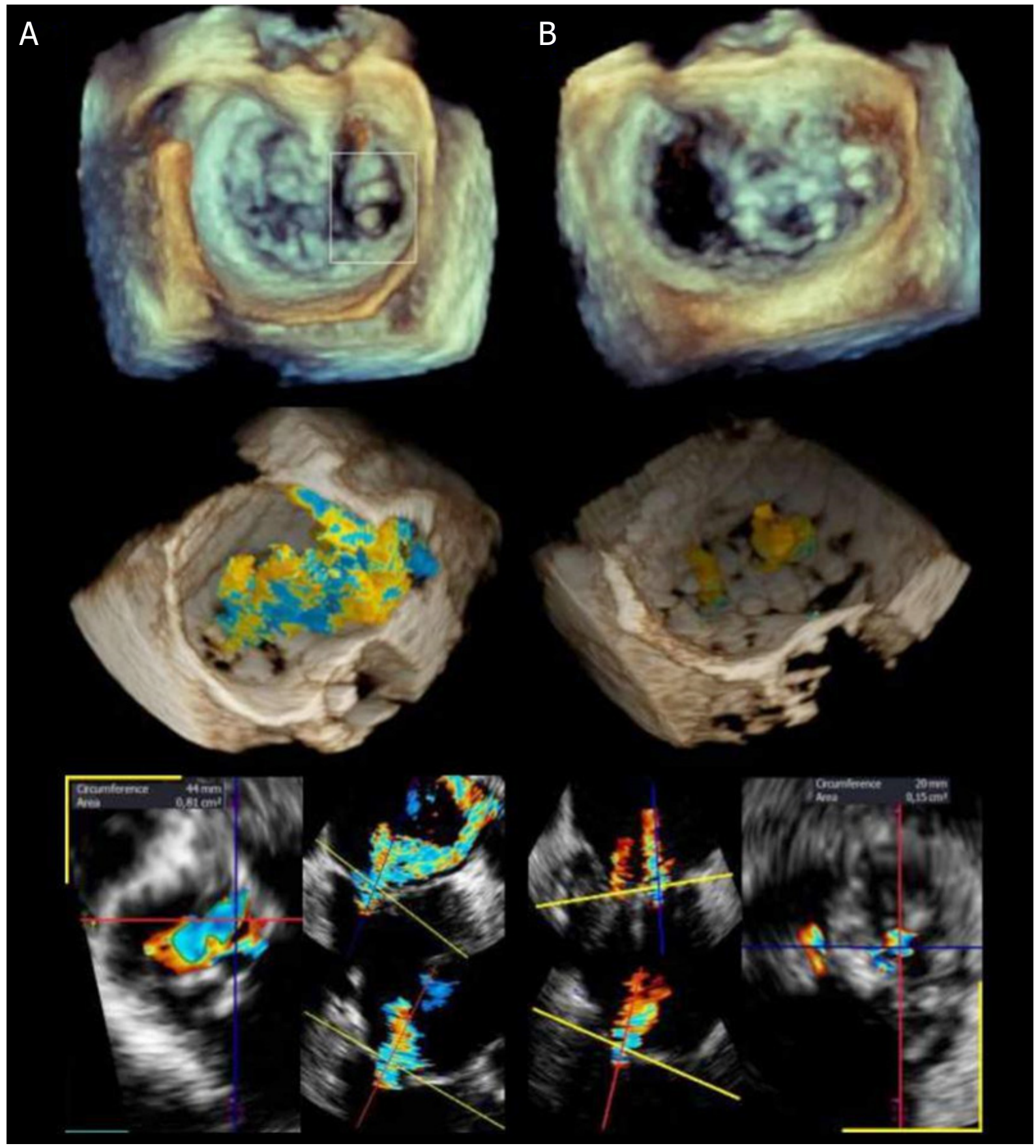

Figure 7. Challenging mitral valve anatomy for percutaneous repair with leaflet approach: commissural large eccentric regurgitant jet originating from P3 prolapse (Column A) effectively treated with the implantation of three clips (Column B)

thickness, calcification, mobility, and measurements in the area of interest [Figure 2]. 3D color-Doppler, especially when using the ventricular perspective, is superior to $2 \mathrm{D}$ color-Doppler to localize the jet with greatest flow convergence area. Measurement of MVA by MPR analysis of 3D dataset is a fundamental step of preprocedural evaluation to avoid significant stenosis after the procedure.

Nowadays, challenging MV anatomies [Figure 7] may be approached in tertiary care centers that recognize only the following as absolute contraindications: a mitral valve area $<3 \mathrm{~cm}^{2}$, calcification in the potential grasping zone [Figure $8 \mathrm{~A}$ ], large cleft extending to the hinge point [Figure $8 \mathrm{~B}$ ], very short $\mathrm{PML}(<7 \mathrm{~mm})$, 

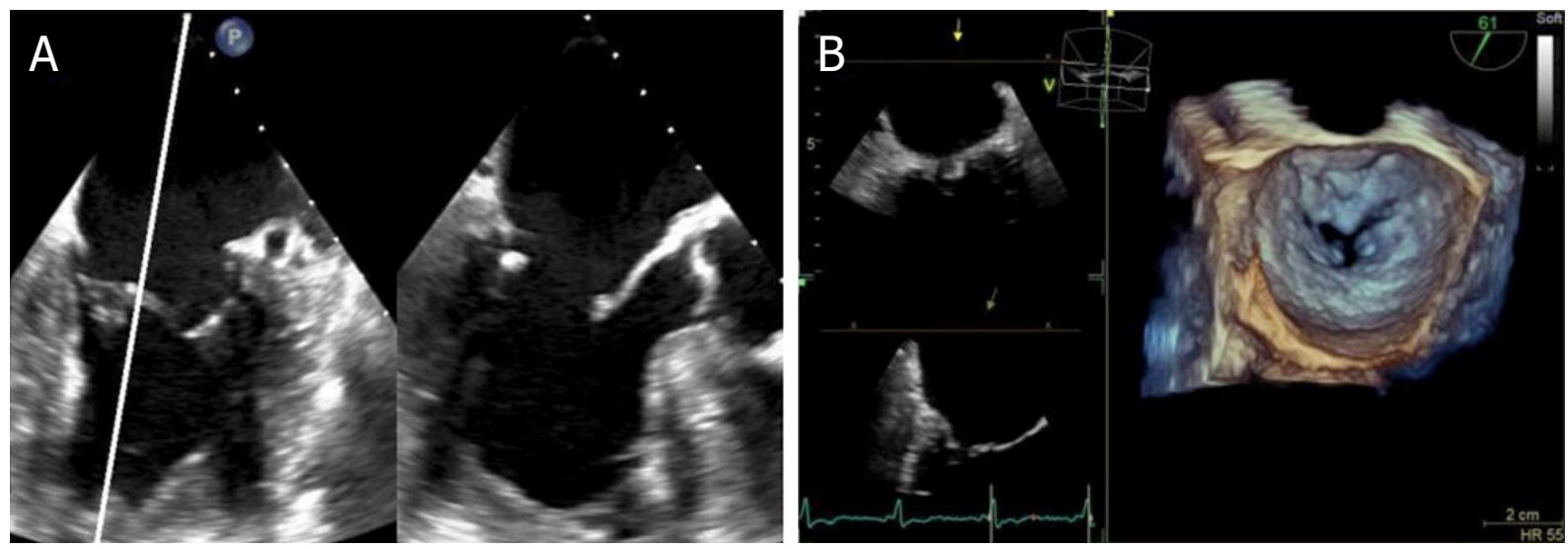

Figure 8. Unsuitable mitral valve anatomy for percutaneous repair with leaflet approach: (A) simultaneous biplane image showing calcification of posterior leaflet in the potential grasping zone; and (B) 3D volume rendering of the mitral valve showing large cleft extending to the hinge point of the posterior leaflet

Table 1. Challenging morphological criteria for percutaneous $\mathrm{MV}$ repair

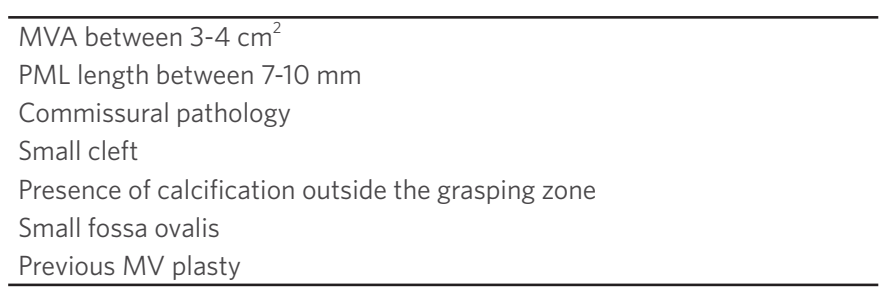

MVA: mitral valve area; PML: posterior mitral leaflet; MV: mitral valve

and endocarditic/rheumatic etiologies or multiple lesions in the context of Barlow disease. Suboptimal MV morphological criteria for performing the procedure are detailed in Table 1. Use of PASCAL (Edwards Lifesciences, USA), thanks to its structural central spacer, may represent a therapeutic option in challenging anatomies (large malcoaptation area, severe annular dilatation, large flail, or prolapse gaps).

The $3 \mathrm{D}$ rendering makes easier the identification of cleft-like indentations, congenital clefts, commissural gaps, and perforations that may represent contraindications to the procedure, but emerging experiences in this setting are promising ${ }^{[37]}$.

Accurate description of the interatrial septum should be performed as the procedure requires a site-specific transseptal puncture in the postero-superior part of the fossa ovalis, $4-4.5 \mathrm{~cm}$ above the MV annulus. Indeed, the presence of a patent foramen ovale, a "floppy" septum, and a small fossa ovalis (FOV) predicts a challenging trans-septal puncture.

For degenerative mitral valve prolapse/flail, the number and identification of diseased segments should be evaluated. The analysis of 3D volumes by MPR can precisely localize [Figure 9] and provide measurement of extension of prolapse/flail (flail gap and flail width, respectively, are ideally $<10 \mathrm{~mm}$ and $<15 \mathrm{~mm}$; a flail width $>10 \mathrm{~mm}$ or high flail gap may predict the need for multiple clips) and discriminate primary and secondary lesion, with the latter possibly left untreated if the principal lesion has been effectively addressed.

In the context of secondary MR, the degree of restriction of posterior leaflet and the degree and extension of coaptation gap (loss of systolic leaflet coaptation in the target zone, easily assessed by $3 \mathrm{D}$ en-face view from LA) should be systematically evaluated. Extension of MV remodeling may be quantified through 

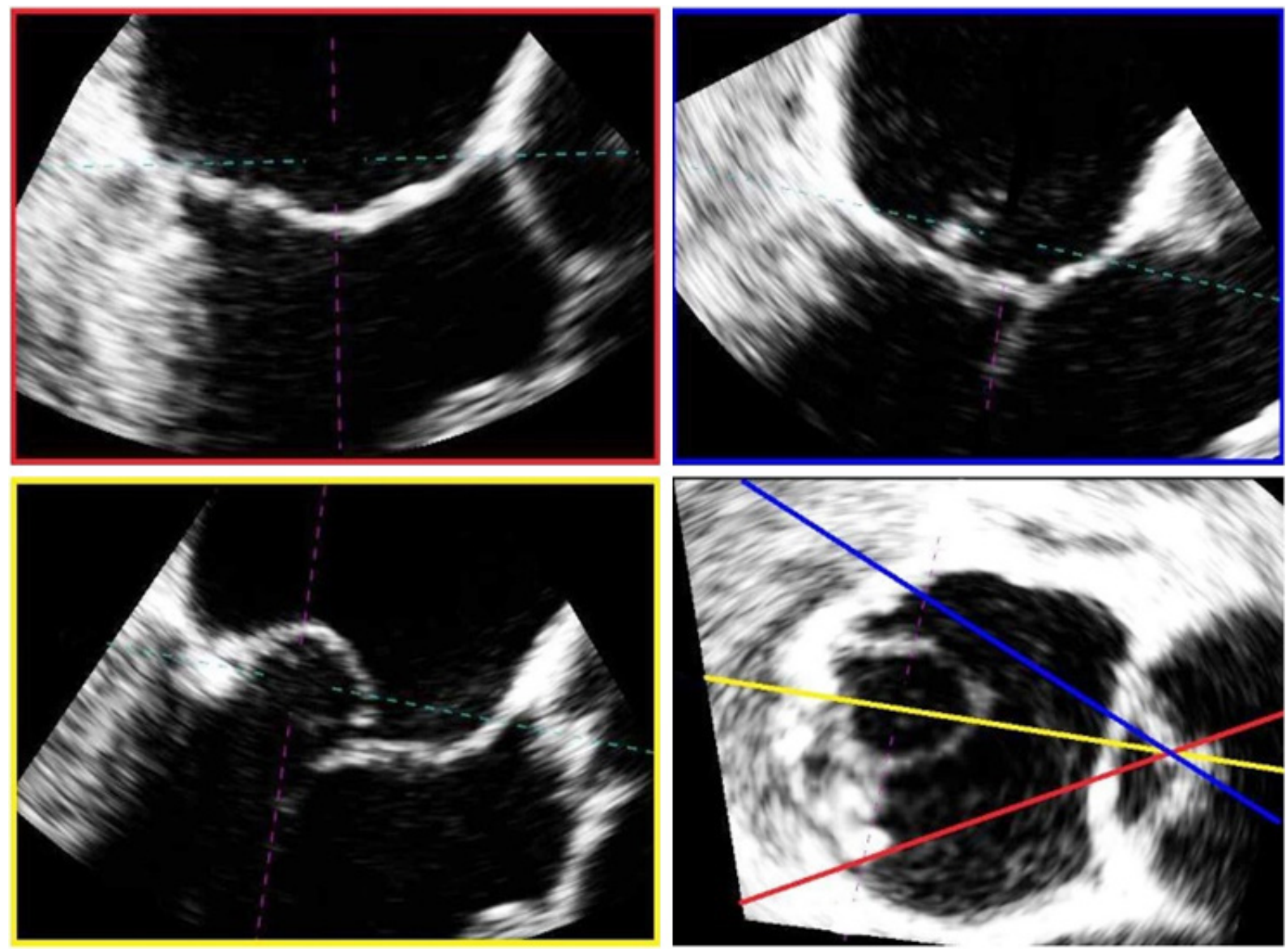

Figure 9. Multiplanar reconstruction analysis of 3D dataset clearly localizing prolapse of the $\mathrm{P} 2$ segment and showing preserved leaflet coaptation in commissural segments

measurement of coaptation depth (distance from the annular plane and the coaptation point, index of tethering) and coaptation length (index of coaptation reserve) that may be assessed in the potential grasping zone by MPR or derived from parametric color-coded maps. Moreover, as contemporary trials have shown, the decision to clip cannot be drawn before a comprehensive evaluation of ventricular dimension and function. Although a unifying, widely accepted explanation for contrasting results of MITRA-FR and COAPT trials has not yet been identified, corroborating evidence suggesting a lack of benefit from the procedure in patients with advanced heart failure is emerging: extreme left ventricular dilation and dysfunction ${ }^{[38,39]}$ and right ventricular dysfunction ${ }^{[40]}$ have been identified as predictors of poor prognosis. The complex interplay between LV geometry and mitral valve function should be taken into account during evaluation for mitral intervention, and EROA/LV end diastolic volume ratio has been proposed to translate it into a measurable variable in order to discriminate patients with features of proportionate or disproportionate $\mathrm{MR}$, with only the latter having survival benefit from the procedure ${ }^{[41]}$. However, concerns on this hypothesis have been raised, relying on accuracy of volume measurements in COAPT trial ${ }^{[42]}$ and highlighting the need for further studies with reliable quantitative measurements for $\mathrm{MR}$ and LV volumes by comprehensive multimodality imaging.

\section{PREPROCEDURAL PLANNING FOR MITRAL VALVE ANNULOPLASTY}

The rationale of surgical annular ring reduction in secondary MR is to improve leaflet apposition, and hence the coaptation reserve, and to prevent the further annular dilatation, without disrupting the mobility of the leaflets. This is achieved through the anterior translation of the posterior annulus, reducing the 
Table 2. Predictors of recurrent MR after isolated surgical MV undersized annuloplasty

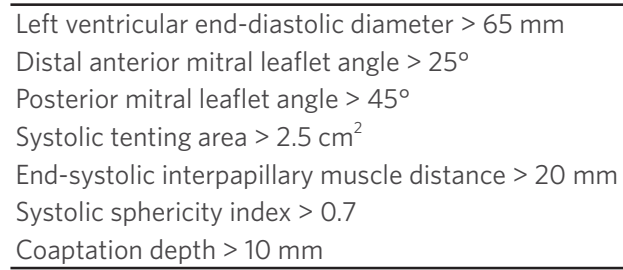

MV: mitral valve; MR: mitral regurgitation

antero-posterior diameter either directly using the Cardioband (Edwards Lifesciences, California, USA) or indirectly with the Carillon device (Cardiac Dimensions, Washington, USA). Secondary MR responsive to annular reduction, according to Carpentier's classification, are Type I (incomplete coaptation due to annular dilatation/deformation, mainly secondary to AF and diastolic dysfunction) and Type IIIb (leaflet tethering with low degree of MV remodeling).

Severe MAC is a contraindication for direct and indirect mitral annuloplasty since it impedes optimal anchoring and contracting of the devices. Surgical experience clearly provides predictors of recurrent MR after isolated undersized annuloplasty ${ }^{[43]}$ [Table 2]. Thus, patient selection is crucial, with ideal candidates being patients with limited leaflet tethering, as suggested by high rate of recurrent MR observed in randomized trials in surgically unselected series ${ }^{[44]}$.

Mitral annulus anatomy as well as relationship with circumflex artery and coronary sinus should be evaluated to assess procedural feasibility and predict procedural success and complications, taking into account the high anatomical variability ${ }^{[45]}$.

The preprocedural planning for direct annuloplasty includes the measurement of the circumference of the posterior MA (from the left to right trigones) at maximum opening of the MV as well as MA area, anteroposterior and medio-lateral diameters, and sphericity index (antero-posterior/mediolateral diameters ratio) obtained preferably by MDCT. MA may also be assessed using echocardiographic 3D dataset by MPR or with dedicated software. The annular thickening should also be measured (minimum desired value is $4 \mathrm{~mm}$ ). MDCT is fundamental to assess the presence, degree, and localization of calcium in the annulus and its extension to the myocardium or leaflet. Noble structures with close relationship with the MA should always be evaluated during the preprocedural planning with dedicated reconstruction by MDCT such as the left circumflex artery (LCX) [Figure 5B], the coronary sinus, and the non-coronary and left coronary aortic cusps adjacent to the base of the anterior leaflet. A minimum distance of $2.5 \mathrm{~mm}$ from the patch of the anchor to the LCX is required to avoid lesion of the artery. Dedicated software based on MDCT can provide the expected intraprocedural fluoroscopic projections and the $3 \mathrm{D}$ preview of the final system position $^{[32,46]}$.

The preprocedural planning of indirect mitral annuloplasty via the coronary sinus requires additional anatomical considerations [Figure 10]. Patency, diameter, tortuosity of the coronary venous system, location and extent of the Thebesian and Vieussens valves, and the spatial relationship of the coronary sinus with respect to the MA and LCX should be evaluated prior the procedure. Distance between the LCX and the coronary sinus must be measured to avoid the coronary impingement during traction of the device. Moreover, to ensure a correct transmission of tension to the annulus, the coronary sinus and MA should lie on the same horizontal plane, information easily obtained by MPR of MDCT dataset. It is quite common for the CS to be located superior to the MA level, thus leading to suboptimal annuloplasty result, because the chincing effect will affect the left atrial wall ${ }^{[47]}$. 

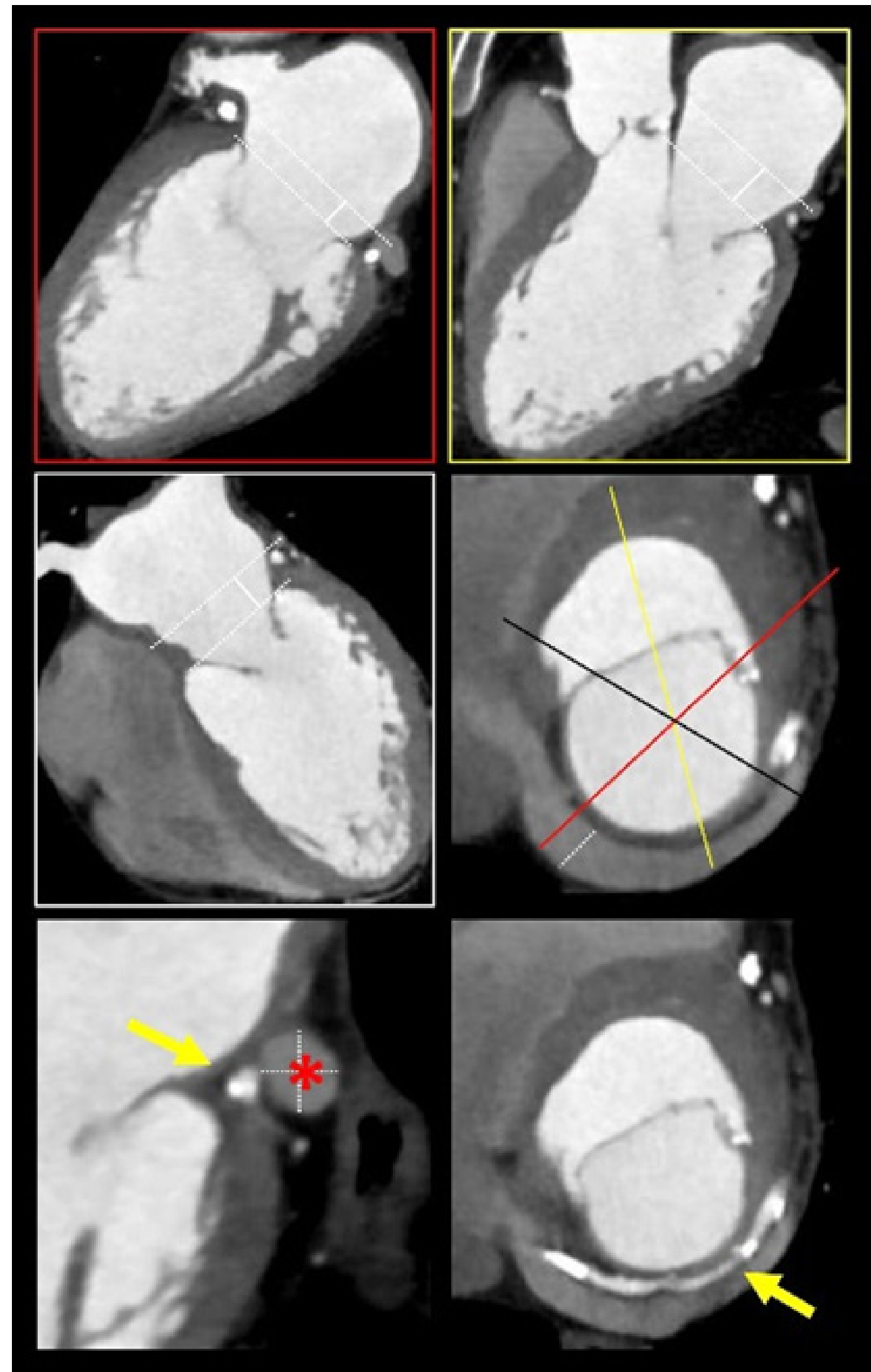

Figure 10. CT preprocedural planning for indirect annuloplasty showing spatial relationship of the coronary sinus with respect to the mitral annulus plane and the LCX (yellow arrow) and CS (red asterisk). LCX: left circumflex artery; CS: coronary sinus 


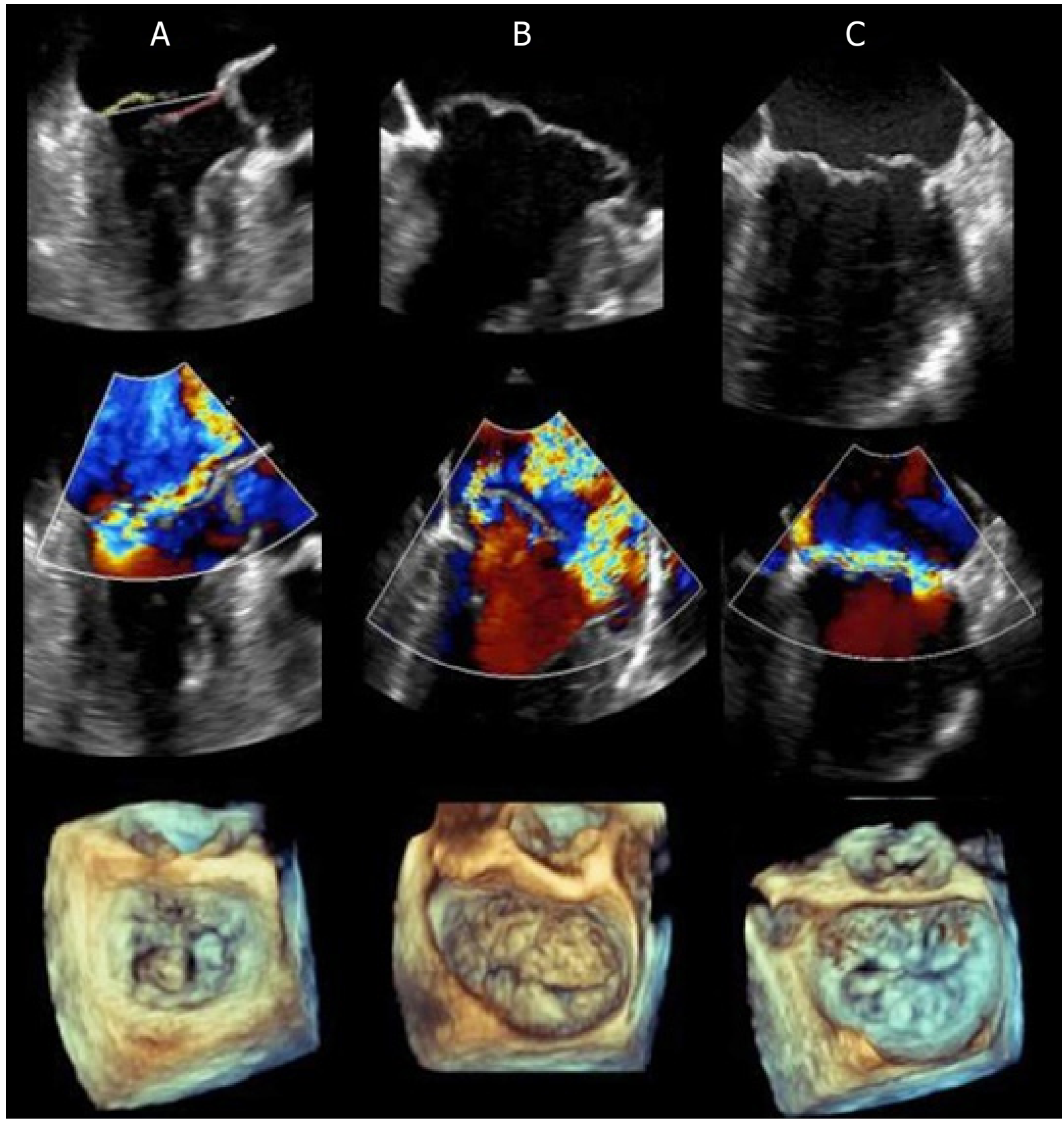

Figure 11. Favorable MV anatomy for chordal implantation: isolated P2 prolapse (A). Unfavorable MV anatomy: (B) bileaflet multiscallop prolapse; and (C) commissural prolapse. MV: mitral valve

Among imaging modalities, invasive venography is the gold standard for the assessment of coronary sinus anatomy; moreover, simultaneous coronary angiography allows assessment of the relation with the coronary arterial tree ${ }^{[48]}$.

\section{PREPROCEDURAL PLANNING FOR MITRAL CHORDAL IMPLANTATION}

Transapical chordal implantation using the NeoChord DS1000 system (NeoChord, Inc., Minnesota, USA) or Harpoon (Edwards Lifescience, California, USA) is a minimally invasive MV repair procedure addressing the correction of isolated prolapse or flail of the posterior leaflet in patients without significant annular dilatation ${ }^{[49]}$. The aims of the preprocedural assessment are: (1) ascertain that patients meet some 
strict anatomical criteria (isolated prolapsed/flail of P2 with a central regurgitant jet); (2) absence of significant annular dilatation; (3) adequate length of the posterior leaflet compared to the antero-posterior MA diameter (ideally $>21 \%$ ); (4) adequate coaptation leaflet reserve; (5) evaluation of MAC because this may cause shadowing and impaired visualization of the device; and (6) determination of the transapical $\operatorname{access}^{[50-52]}$ [Figure 11].

Transthoracic echocardiography is frequently used to decide the optimal intercostal space and location for the mini-thoracotomy. MDCT permits the visualization of the anatomical relation between apex and chest wall and the definition of a trajectory for the device.

\section{CONCLUSION}

Transcatheter mitral valve interventions provide a new spectrum of therapeutic options for high-risk patients. Accurate patient selection and choice of the treatment strategy, either leaflet or annular approach, or combined, goes through a comprehensive preprocedural multimodality imaging evaluation aimed at the characterization of the functional anatomy of MR and its interplay with left ventricular geometry and function.

\section{DECLARATIONS}

\section{Authors' contributions}

Proof writing: Melillo F, Boccellino A

Proof revision: Ingallina G, Ancona F, Capogrosso C, Napolano A, Stella S, Agricola E

Conception and design: Melillo F, Agricola E

\section{Availability of data and materials}

Not applicable.

\section{Financial support and sponsorship}

None.

\section{Conflicts of interest}

All authors declared that there are no conflicts of interest.

\section{Ethical approval and consent to participate}

Not applicable.

\section{Consent for publication}

Not applicable.

\section{Copyright}

(c) The Author(s) 2020.

\section{REFERENCES}

1. Del Forno B, De Bonis M, Agricola E, et al. Mitral valve regurgitation: a disease with a wide spectrum of therapeutic options. Nat Rev Cardiol 2020:1-21.

2. Zekry SB, Nagueh SF, Little SH, et al. Comparative accuracy of two- and three-dimensional transthoracic and transesophageal echocardiography in identifying mitral valve pathology in patients undergoing mitral valve repair: initial observations. $J$ Am Soc Echocardiogr 2011;24:1079-85.

3. Faletra FF, Leo LA, Paiocchi VL, et al. Anatomy of mitral annulus insights from non-invasive imaging techniques. Eur Heart $J$ Cardiovasc Imaging 2019;20:843-57.

4. Heo R, Son JW, Hartaigh BÓ, et al. Clinical Implications of Three-Dimensional Real-Time Color Doppler Transthoracic 
Echocardiography in Quantifying Mitral Regurgitation: A Comparison with Conventional Two-Dimensional Methods. J Am Soc Echocardiogr 2017;30:393-403.

5. Nolan MT, Thavendiranathan P. Automated Quantification in Echocardiography. JACC Cardiovasc Imaging 2019;12:1073-92.

6. Dorosz JL, Lezotte DC, Weitzenkamp DA, Allen LA, Salcedo EE. Performance of 3-dimensional echocardiography in measuring left ventricular volumes and ejection fraction: a systematic review and meta-analysis. J Am Coll Cardiol 2012;59:1799-808.

7. Papadopoulos K, Ikonomidis I, Chrissoheris M, et al. MitraClip and left ventricular reverse remodelling: a strain imaging study. ESC Heart Fail 2020;7:1409-18.

8. Baumgartner H, Falk V, Bax JJ, et al; ESC Scientific Document Group. 2017 ESC/EACTS Guidelines for the management of valvular heart disease. Eur Heart $J$ 2017;38:2739-91.

9. Zoghbi WA, Adams D, Bonow RO, et al. Recommendations for Noninvasive Evaluation of Native Valvular Regurgitation: A Report from the American Society of Echocardiography Developed in Collaboration with the Society for Cardiovascular Magnetic Resonance. $J \mathrm{Am}$ Soc Echocardiogr 2017;30:303-71.

10. Zeng X, Levine RA, Hua L, et al. Diagnostic value of vena contracta area in the quantification of mitral regurgitation severity by color Doppler 3D echocardiography. Circ Cardiovasc Imaging 2011;4:506-13.

11. Bartko PE, Arfsten H, Heitzinger G, et al. A Unifying Concept for the Quantitative Assessment of Secondary Mitral Regurgitation. $J$ Am Coll Cardiol 2019;73:2506-17.

12. Lancellotti P, Magne J, Dulgheru R, Ancion A, Martinez C, Piérard LA. Clinical significance of exercise pulmonary hypertension in secondary mitral regurgitation. Am J Cardiol 2015;115:1454-61.

13. Lancellotti P, Gérard PL, Piérard LA. Long-term outcome of patients with heart failure and dynamic functional mitral regurgitation. Eur Heart $J$ 2005;26:1528-32.

14. Velu JF, Baan J Jr, de Bruin-Bon HACM, et al. Can stress echocardiography identify patients who will benefit from percutaneous mitral valve repair? Int J Cardiovasc Imaging 2019;35:645-51.

15. Uretsky S, Argulian E, Narula J, Wolff SD. Use of Cardiac Magnetic Resonance Imaging in Assessing Mitral Regurgitation: Current Evidence. J Am Coll Cardiol 2018;71:547-63.

16. Hundley WG, Li HF, Willard JE, et al. Magnetic resonance imaging assessment of the severity of mitral regurgitation. Comparison with invasive techniques. Circulation 1995;92:1151-8.

17. Uretsky S, Gillam L, Lang R, et al. Discordance between echocardiography and MRI in the assessment of mitral regurgitation severity: a prospective multicenter trial. J Am Coll Cardiol 2015;65:1078-88.

18. Fujita N, Chazouilleres AF, Hartiala JJ, et al. Quantification of mitral regurgitation by velocity-encoded cine nuclear magnetic resonance imaging. Journal of the American College of Cardiology 1994;23:951-8.

19. Gelfand EV, Hughes S, Hauser TH, et al. Severity of mitral and aortic regurgitation as assessed by cardiovascular magnetic resonance: optimizing correlation with Doppler echocardiography. J Cardiovasc Magn Reson 2006;8:503-7.

20. Lopez-Mattei JC, Ibrahim H, Shaikh KA, et al. Comparative Assessment of Mitral Regurgitation Severity by Transthoracic Echocardiography and Cardiac Magnetic Resonance Using an Integrative and Quantitative Approach. Am J Cardiol 2016;117:264-70.

21. Sachdev V, Hannoush H, Sidenko S, et al. Are Echocardiography and CMR Really Discordant in Mitral Regurgitation? JACC Cardiovasc Imaging 2017;10:823-4.

22. Choi J, Heo R, Hong GR, et al. Differential effect of 3-dimensional color Doppler echocardiography for the quantification of mitral regurgitation according to the severity and characteristics. Circ Cardiovasc Imaging 2014;7:535-44.

23. Thavendiranathan P, Liu S, Datta S, et al. Quantification of chronic functional mitral regurgitation by automated 3-dimensional peak and integrated proximal isovelocity surface area and stroke volume techniques using real-time 3-dimensional volume color Doppler echocardiography: in vitro and clinical validation. Circ Cardiovasc Imaging 2013;6:125-33.

24. Westenberg JJ, Roes SD, Marsan NA, et al. Mitral valve and tricuspid valve blood flow: accurate quantification with 3D velocity-encoded MR imaging with retrospective valve tracking. Radiology 2008;249:792-800.

25. Garg P, Swift AJ, Zhong L, et al. Assessment of mitral valve regurgitation by cardiovascular magnetic resonance imaging. Nat Rev Cardiol 2020;17:298-312.

26. Myerson SG, d'Arcy J, Christiansen JP, et al. Determination of Clinical Outcome in Mitral Regurgitation With Cardiovascular Magnetic Resonance Quantification. Circulation 2016;133:2287-96.

27. Penicka M, Vecera J, Mirica DC, Kotrc M, Kockova R, Camp GV. Prognostic Implications of Magnetic Resonance-Derived Quantification in Asymptomatic Patients With Organic Mitral Regurgitation: Comparison With Doppler Echocardiography-Derived Integrative Approach. Circulation 2018;137:1349-60.

28. Cavalcante JL, Kusunose K, Obuchowski NA, et al. Prognostic Impact of Ischemic Mitral Regurgitation Severity and Myocardial Infarct Quantification by Cardiovascular Magnetic Resonance. JACC Cardiovasc Imaging 2020;13:1489-501.

29. Marra MP, Basso C, De Lazzari M, et al. Morphofunctional Abnormalities of Mitral Annulus and Arrhythmic Mitral Valve Prolapse. Circ Cardiovasc Imaging 2016;9:e005030.

30. Miller MA, Dukkipati SR, Turagam M, Liao SL, Adams DH, Reddy VY. Arrhythmic mitral valve prolapse: JACC review topic of the week. J Am Coll Cardiol 2018;72:2904-14.

31. Rowin EJ, Maron BJ, Maron MS. The hypertrophic cardiomyopathy phenotype viewed through the prism of multimodality imaging: clinical and etiologic implications. JACC Cardiovasc Imaging 2020;13:2002-16.

32. Faggioni L, Gabelloni M, Accogli S, et al. Preprocedural planning of transcatheter mitral valve interventions by multidetector CT: what the radiologist needs to know. Eur J Radiol Open 2018;5:131-40. 
33. Khalique OK, Hahn RT. Multimodality imaging in transcatheter mitral interventions: buzzword or modern age toolbox? Circ Cardiovasc Imaging 2016;9:e005071.

34. Kim SS, Ko SM, Song MG, et al. Quantification of stenotic mitral valve area and diagnostic accuracy of mitral stenosis by dualsource computed tomography in patients with atrial fibrillation: comparison with cardiovascular magnetic resonance and transthoracic echocardiography. Int J Cardiovasc Imaging 2015;31 Suppl 1:103-14.

35. van Rosendael PJ, van Wijngaarden SE, Kamperidis V, et al. Integrated imaging of echocardiography and computed tomography to grade mitral regurgitation severity in patients undergoing transcatheter aortic valve implantation. Eur Heart J 2017;38:2221-6.

36. Alfieri O, Maisano F, De Bonis M, et al. The double-orifice technique in mitral valve repair: a simple solution for complex problems. $J$ Thorac Cardiovasc Surg 2001;122:674-81.

37. Melillo E, Ancona F, Buzzatti N, Denti P, Agricola E. A challenging mitral valve anatomy for percutaneous repair with MitraClip: cleft posterior leaflet. Eur Heart J Cardiovasc Imaging 2019;20:1433-4.

38. Puls M, Lubos E, Boekstegers P, et al. One-year outcomes and predictors of mortality after MitraClip therapy in contemporary clinical practice: results from the German transcatheter mitral valve interventions registry. Eur Heart J 2016;37:703-12.

39. Godino C, Scotti A, Taramasso M, et al. Two-year cardiac mortality after MitraClip treatment of functional mitral regurgitation in ischemic and non-ischemic dilated cardiomyopathy. Int J Cardiol 2018;269:33-9.

40. Kaneko H, Neuss M, Weissenborn J, Butter C. Prognostic Significance of Right Ventricular Dysfunction in Patients With Functional Mitral Regurgitation Undergoing MitraClip. Am J Cardiol 2016;118:1717-22.

41. Grayburn PA, Sannino A, Packer M. Proportionate and Disproportionate Functional Mitral Regurgitation: A New Conceptual Framework That Reconciles the Results of the MITRA-FR and COAPT Trials. JACC Cardiovasc Imaging 2019;12:353-62.

42. Gaasch WH, Aurigemma GP, Meyer TE. An Appraisal of the Association of Clinical Outcomes With the Severity of Regurgitant Volume Relative to End-Diastolic Volume in Patients With Secondary Mitral Regurgitation. JAMA Cardiol 2020;5:476-81.

43. Ciarka A, Braun J, Delgado V, et al. Predictors of mitral regurgitation recurrence in patients with heart failure undergoing mitral valve annuloplasty. Am J Cardiol 2010;106:395-401.

44. Goldstein D, Moskowitz AJ, Gelijns AC, et al; CTSN. Two-year outcomes of surgical treatment of severe ischemic mitral regurgitation. $N$ Engl J Med 2016;374:344-53.

45. Spencer JH, Prahl G, Iaizzo PA. The prevalence of coronary sinus and left circumflex artery overlap in relation to the mitral valve. $J$ Interv Cardiol 2014;27:308-16.

46. Bax JJ, Debonnaire P, Lancellotti P, et al. Transcatheter interventions for mitral regurgitation: multimodality imaging for patient selection and procedural guidance. JACC Cardiovasc Imaging 2019;12:2029-48.

47. Tops LF, Van de Veire NR, Schuijf JD, et al. Noninvasive evaluation of coronary sinus anatomy and its relation to the mitral valve annulus: implications for percutaneous mitral annuloplasty. Circulation 2007;115:1426-32.

48. Schofer J, Siminiak T, Haude M, et al. Percutaneous mitral annuloplasty for functional mitral regurgitation: results of the CARILLON Mitral Annuloplasty Device European Union Study. Circulation 2009;120:326-33.

49. Seeburger J, Rinaldi M, Nielsen SL, et al. Off-pump transapical implantation of artificial neo-chordae to correct mitral regurgitation: the TACT Trial (Transapical Artificial Chordae Tendinae) proof of concept. J Am Coll Cardiol 2014;63:914-9.

50. Gammie JS, Wilson P, Bartus K, et al. Transapical beating-heart mitral valve repair with an expanded polytetrafluoroethylene cordal implantation device: initial clinical experience. Circulation 2016;134:189-97.

51. Rucinskas K, Janusauskas V, Zakarkaite D, et al. Off-pump transapical implantation of artificial chordae to correct mitral regurgitation: early results of a single-center experience. J Thorac Cardiovasc Surg 2014;147:95-9.

52. Colli A, Manzan E, Zucchetta F, et al. Transapical off-pump mitral valve repair with Neochord implantation: Early clinical results. Int $J$ Cardiol 2016;204:23-8. 